\title{
INTELIGENCIA ARTIFICIAL Y/O EL ARQUITECTO
}

\author{
ARTIFICIAL INTELLIGENCE \\ AND/OR THE ARCHITECT
}

FAVIO CHUMPITAZ REQUENA

Universidad de Lima

Los avances y el desarrollo de la tecnología en distintas herramientas, software y hardware, generan grandes implicancias en el proceso de la concepción del diseño arquitectónico. Dentro de ella, la inteligencia artificial puede marcar un punto de quiebre en la relación entre las máquinas y los arquitectos. Al brindarle a las máquinas una mayor capacidad de inteligencia, razonamiento y sensibilidad, los arquitectos podrán analizar con mayor profundidad un programa arquitectónico, pudiendo producir una gran cantidad de ideas y variables en las propuestas, tomando incluso distintos lenguajes arquitectónicos previamente aprendidos y modificando su propia labor en el proceso de diseño.

inteligencia artificial, arquitectura, diseño, lenguaje arquitectónico, arquitecto
Recibido: 3 de junio del 2019

Aprobado: 6 de agosto del 2019

doi: 10.26439/limaq2020.n006.4820

Technological advances and development in different tools, software and hardware affect, to a great extent, the process of conceiving architectural designs. In technology, artificial intelligence can mark a turning point in the relationship between machines and architects. By giving machines greater capacity of intelligence, reasoning and sensibility, architects will be able to analyze more deeply an architectural program, and produce a large number of ideas and variables in their proposal, even considering different previously learned architectural languages, and varying their work in the design process.

artificial intelligence, architecture, design, architectural language, architect 


\section{INTRODUCCIÓN}

En diversos ámbitos profesionales, el desarrollo de la tecnología y sus aplicaciones han tenido un gran impacto. En ese sentido, la arquitectura no es ni puede ser ajena al proceso en curso; por el contrario, el avance de la tecnología y su desarrollo ha llevado a facilitar la labor de los arquitectos en distintos aspectos.

En la etapa de concepción del diseño de un proyecto, la tecnología ha permitido a los proyectistas poseer mayores herramientas para la producción de modelos que muestren sus ideas y generen sus múltiples opciones, en menor tiempo, con mejor precisión y calidad. Esto les ha permitido ampliar las posibilidades de creación al poder decidir sobre una mayor cantidad de opciones producidas.

Esto es posible, no solo por el desarrollo y mejoramiento constante de nuevos hardwares y softwares que permiten una mejor capacidad de almacenamiento, velocidad de funcionamiento y mejor interfaz, sino sobre todo por un mejor procesamiento y razonamiento de la información, la cual intenta emular a la de los humanos.

Esta intuición y/o emulación con la que se programa y desarrolla a las máquinas, principalmente en sus softwares, busca reproducir o imitar diversas características que se catalogan como propias de los humanos, tales como la sensibilidad e intuición, pensamiento y raciocinio, con el fin de que puedan generar una respuesta frente a cualquier problema planteado y ante cualquier situación inesperada, siendo conscientes del entorno inmediato en el cual están interactuando. Emulando en ese actuar, la sensibilidad e intuición del ser humano.

\section{INTELIGENCIA ARTIFICIAL}

Esta búsqueda de emular el razonamiento de los humanos empezó como un proyecto de investigación de verano a cargo de John $\mathrm{McCarthy}^{1}$, profesor de Dartmouth en Estados Unidos, quien, junto con un grupo de investigadores, a mediados de la década del 50, reflexionaron sobre cómo implementar y desarrollar en las máquinas el uso del lenguaje, buscando un nivel similar a los humanos; sin embargo, el trabajo no tuvo el éxito esperado.

Luego de ello, otros investigadores se enfocaron más en desarrollar algoritmos que pudieran resolver problemas lógicos y matemáticos; no obstante, fue a fines de la década del 50 que el investigador Arthur Samuel ${ }^{2}$ desarrolla un programa capaz de aprender y mejorar su propio desempeño al jugar damas chinas. Esto convertiría al juego en el primero en ser entendido por una

1 John McCarthy (1927-2011) científico informático, ganador del premio Turing de 1971, desarrollo el lenguaje de programación LISP y fue uno de los fundadores del campo de la inteligencia artificial.

2 Arthur Samuel (1901-1990) fue pionero en el campo de la inteligencia artificial al realizar una demostración temprana de esta a través del diseño de su programa de juego. 
máquina que es capaz de analizar profundamente las probabilidades que se tienen al mover las fichas. De este modo, se logró el denominado aprendizaje de memoria rote learning, con el que dicho programa fue capaz de vencer, al menos en una partida, al maestro de las damas, Robert Nealey, en 1962.

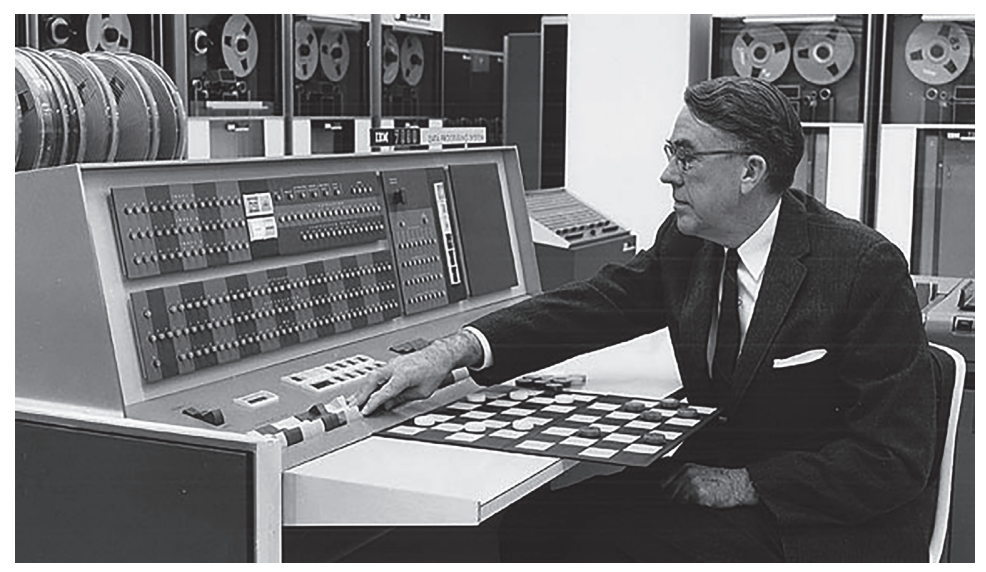

Figura 1.

Arthur Samuel

jugando damas

con la máquina

IBM 701

Fuente: IBM

https://www.ibm. com/ibm/history/

ibm100/us/en/

icons/ibm700se-

ries/impacts/

El siguiente paso importante en el desarrollo de la inteligencia artificial (IA) se le atribuye a Joseph Weizenbaum ${ }^{3}$ del MIT (Massachusetts Institute of Technology) en 1965, considerado uno de los padres de la inteligencia artificial moderna, quien desarrolla el primer programa de comunicación en lenguaje natural-llamado "Eliza" - que simulaba una conversación entre la máquina con el humano a partir de una comparación de patrones.

Luego de ello, existieron diversos aportes importantes entre los que destaca Meta-Dentral, en 1975, que se convirtió en el primer programa en generar un contenido a partir de su interpretación de un análisis químico.

En 1987, Ernst Dickmanns desarrollo el soporte de un prototipo de vehículo de manejo automático usando para ello varias computadoras y cámaras a fin de procesar lo que ocurría a su alrededor. La unidad logró autoconducirse a lo largo de veinte kilómetros por una pista alemana.

En 1997 la empresa de tecnología IBM desarrolla Deep Blue, solución tecnológica que podía analizar con mayor complejidad y proyección el desarrollo del ajedrez, a fin de decidir sus movimientos. Este programa fue capaz de derrotar al campeón mundial de ajedrez, Gary Kasparov.

Pero no fue hasta el 2016 en donde se produjo uno de los principales logros de la IA bajo la creación de Google DeepMind": AlphaGo. Este programa fue capaz

3 Joseph Weizenbaum (1923-2008) fue un científico informático y profesor del MIT, autor del libro: Computer Power and Human Reason: From Judgment to Calculation (1976).

4 DeepMind, tiene como misión científica ampliar los límites de la inteligencia artificial. 
Figura 2

Lee Sedol versus

AlphaGo

Fuente: Wired

https://

www.wired.

com/2016/05/

google-alpha-

go-ai/

de entender, intuir y dominar el estratégico y complejo juego de mesa Go, que tiene más de 3000 años de antigüedad y cuyo objetivo es controlar la mayor cantidad del área del tablero rodeándolo con las fichas. Las variables que posee a diferencia de otros juegos de mesa son abismales debido a la complejidad respecto de colocar las piezas y los movimientos dentro del tablero; mientras que al jugar ajedrez se tiene en un turno treinta y cinco opciones de elegir un movimiento, en Go son cerca de doscientas opciones. Como describe Metz (2016) en su texto: "El ajedrez es una metáfora de la guerra, pero es solo una simple batalla; Go es como un espacio de batalla global" .

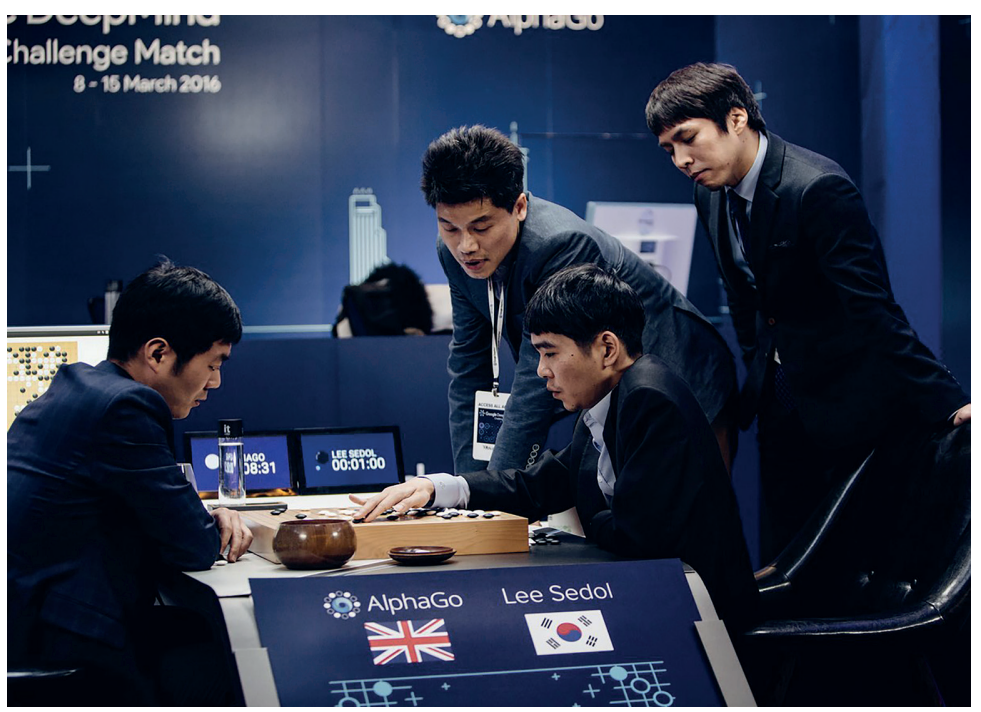

Este nivel de compresión de AlphaGo fue posible gracias al desarrollo de sus algoritmos a nivel del autoaprendizaje, lo que le permitió aprender de sus propios errores al jugar contra sí mismo, y también a través de las partidas jugadas contra humanos, entre ellos, el campeón europeo Fan Hui.

Finalmente, AlphaGo derrotaría al campeón mundial Lee Sedol, considerado el héroe nacional de Corea del Sur, venciéndolo en cuatro juegos de cinco, lo que se volvió un suceso mundial al creer inconcebible que una máquina pudiera no solo aprender el complejo juego Go, sino, sobre todo, al momento de jugar, intuir como un humano y plantear nuevos movimientos a partir de ello.

Como declaró Metz (2016) luego de haber concluido el esperado encuentro contra el campeón mundial:

5 "Chess is a metaphor for war, but it's really just a single battle. Go is like a global battlespace" (traducción del autor). 
AlphaGo mostró lo que los jugadores de Go podrían describir como intuición, la capacidad de jugar un juego hermoso no solo como una persona, sino de una manera en que ninguna persona podría ${ }^{6}$.

AlphaGo, en cierto sentido, comenzó a pensar por sí mismo. Tomaba decisiones basadas no en un conjunto de reglas que sus creadores habían codificado en su ADN digital, sino en los algoritmos que se habían enseñado a sí mismos. Realmente descubrió esto por sí mismo, a través de su propio proceso de introspección y análisis?

La máquina hizo algo muy humano incluso mejor que un humano. Pero en el proceso hizo a esos humanos mejores en lo que hacen. Sí, podrías ver el movimiento 37 como un signo temprano de máquinas que afirman su superioridad sobre sus creadores humanos. O podrías verlo como una semilla: sin movimiento 37 , no tendríamos movimiento $78^{8}$.

Fue el momento en que AlphaGo demostró que entiende, o al menos parece imitar la comprensión de una manera que no se puede distinguir de la realidad 9 .

La complejidad del Go era tal que incluso para una computadora de gran capacidad era imposible considerar todos los resultados posibles por cada movimiento que realizaba. Por ello, se buscaba que AlphaGo pudiera elaborar sus propias propuestas para aprender a jugar razonablemente, alimentándola con cerca de 30 millones de movimientos del Go en su red neuronal, una red que imitaba las neuronas del cerebro humano. El movimiento $37^{10}$ realizado por AlphaGo no se encontraba dentro de esos 30 millones, sino que fue una decisión analizada por la máquina y propuesta por ella, creando así su propio repertorio de jugadas.

Es ese nivel de imitación alcanzado por Alpha Go -de pensar o actuar como humano de manera racional- lo que define o ejemplifica mejor a la inteligencia artificial. En cuanto a una definición de este concepto, los científicos especialistas en el tema, Stuart Russell y Peter Norvig, autores del libro Inteligencia artificial: un enfoque moderno (1995), describen cómo ha sido definido, a lo largo del tiempo, bajo estos cuatro grupos: sistemas que piensan como humanos, sistemas que piensan racionalmente, sistemas que actúan como humanos y sistemas que actúan racionalmente.

6 "AlphaGo displayed what Go players might describe as intuition, the ability to play a beautiful game not just like a person but in a way no person could" (traducción del autor).

7 "AlphaGo had, in a sense, started to think on its own. It was making decisions based not on a set of rules its creators had encoded in its digital DNA but on algorithms it had taught itself. It really discovered this for itself, through its own process of introspection and analysis" (traducción del autor).

8 "The machine did a very human thing even better than a human. But in the process it made those humans better at what they do. Yes, you could see Move 37 as an early sign of machines asserting their superiority to their human creators. Or you could see it as a seed: Without Move 37, we wouldn't have Move 78" (traducción del autor)

9 "It was the moment AlphaGo proved it understands, or at least appears to mimic understanding in a way that is indistinguishable from the real thing" (traducción del autor).

10 Movimiento 37 de AlphaGo, en el segundo juego contra Lee Sedol fue considerado como único. 
Es entonces que este desarrollo constante y evolutivo de la inteligencia artificial ha llevado a distintas disciplinas, entre ellas a la arquitectura, a cuestionarse respecto de su alcance, beneficios y criterios de uso, pues si bien los arquitectos se han visto beneficiados por programas para modelar y procesar información que permita elaborar un proyecto desde hace ya cincuenta años, esta nueva y futura relación entre la inteligencia artificial y el arquitecto podría ser distinta.

\section{Y/O EL ARQUITECTO}

Matthias Kohler ${ }^{11}$ (2018), profesor de arquitectura y fabricación digital de la ETH Zurich, considera que la arquitectura va a seguir cambiando de la misma manera que lo hace cuando utiliza nuevos materiales o sistemas constructivos. Además estima que el uso de la tecnología va a permitir distintas formas de experimentar y vivir cada edificación. Por lo que el diseño se volverá mucho más colaborativo manteniendo siempre la premisa de que su elección sea realizada por un profesional en el tema, diseñador o arquitecto.

Respecto a la posibilidad de que las máquinas, a través del desarrollo de sus algoritmos, tomaran la posta de los arquitectos, Kohler reflexiona que es una idea que podría darse en el futuro, aunque la considera poco probable por la falta de interacción que puede tener una máquina con un cliente. Dicha interacción constituye una pieza fundamental del trabajo creativo que se sustenta en la intuición y sensibilidad del arquitecto para diseñar una nueva propuesta. Además los describe como puntos que no podrían ser realizados por un algoritmo o máquina con inteligencia artificial, ya que esta requeriría de la sensibilidad humana para entender contextos sociales, anhelos del cliente y distintas particularidades que se dan en el proceso del diseño de un nuevo proyecto. El profesor recalca en una entrevista: "No creo que la inteligencia artificial sustituya a los arquitectos, el diseño es más social” (Koller 2018).

En esa misma línea de ideas, el investigador Imdat $\mathrm{As}^{12}$ reflexiona sobre el impacto que tiene la inteligencia artificial en la arquitectura describiendo que actualmente se está viviendo un punto de inflexión en la historia al permitir que esta pueda expandir nuestros conocimientos en torno al diseño en aspectos que no conocemos o que no somos capaces de conectar o producir. Desde el año 2011, el impulso dado al desarrollo de la investigación en DNN (Deep Neural Network), que imita el funcionamiento interno del cerebro humano, poseyendo distintas capas neuronales, ha permitido almacenar una gran cantidad de información, procesarla, analizarla y evaluarla a partir de lo aprendido bajo distintos criterios.

En lo referido a la arquitectura, uno de los diseños de DNN fue capaz de calificar la habitabilidad de diseños de espacios bajo los criterios brindados por

11 Matthias Kohler (1968) es un arquitecto especialista en diseño computacional, fabricación robótica e innovación de materiales.

12 Imdat As, arquitecto especialista en diseño digital e investigador. 

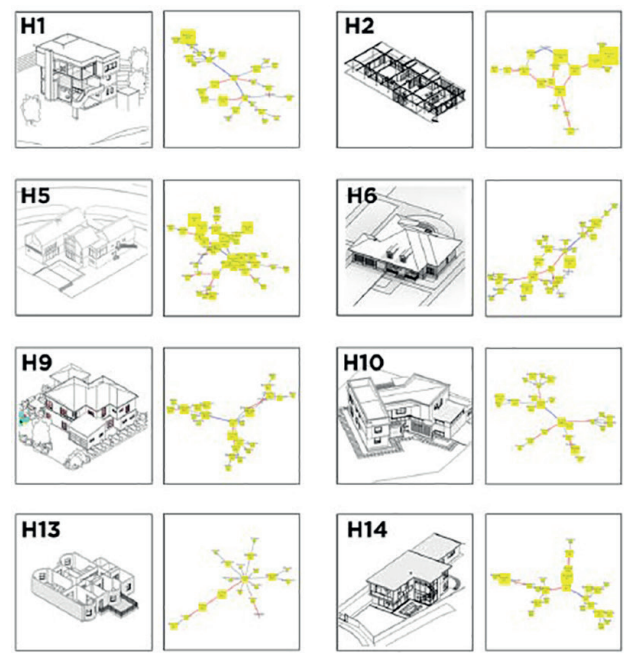

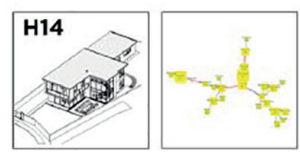

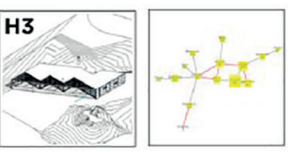
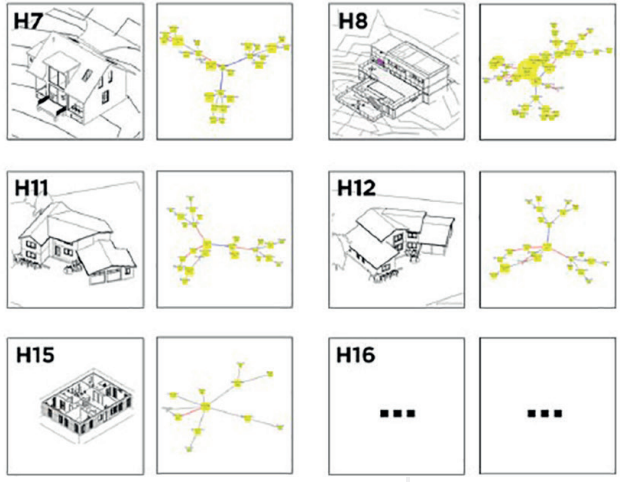

Figura 3.

Viviendas usa-

das de ejemplo

para analizar su

habitabilidad

Fuente: As, Pal,

y Basu, 2018,

p. 315 en el terreno. Esto es posible al tener mucha mejor claridad y capacidad para analizar, identificar y clasificar toda la información disponible que exista del terreno, del programa arquitectónico y del diseño requerido con base en sus funciones. También para decodificar patrones en el diseño arquitectónico que no necesariamente sean tangibles, a fin de que el arquitecto pueda optar por decisiones mucho más inteligentes permitiendo un nuevo tipo de composición y/o diseño. Ejemplo: el DNN podría encontrar patrones para diseñar un espacio de carácter espiritual al revisar y analizar miles de ejemplos de este tipo para descifrar patrones como las proporciones de los espacios, la iluminación, altura, etcétera, siendo algunos de estos datos obvios, pero otros quizá nunca pensados. De igual manera, se podrían tomar los lenguajes de patrones del diseño residencial de Christopher Alexander y potenciarlo y expandirlo para llevarlo a otro nivel.

Otro claro ejemplo del impacto positivo es la suma de plataformas adicionales de programación a los programas de diseño para que se alimenten entre sí brindando mayores posibilidades para generar opciones volumétricas como es el caso del programa de diseño Rhinoceros ${ }^{13}$ en conjunto con el programa

13 Rhinoceros es un programa de modelación 3D. 
Grasshopper ${ }^{14}$. La combinación de ambos, hoy en día, es capaz de brindarnos múltiples opciones a partir de los parámetros que el usuario les asigne. Si tomamos este referente actual como muestra del desarrollo potencial que posee la inteligencia artificial y su constante búsqueda de lograr que las máquinas puedan evolucionar hasta alcanzar una inteligencia, racionalidad e intuición mayor a la de los humanos, es posible imaginar que en el futuro, y como ha ocurrido en la historia, las máquinas puedan ir suplantando a los humanos en nuevas labores.
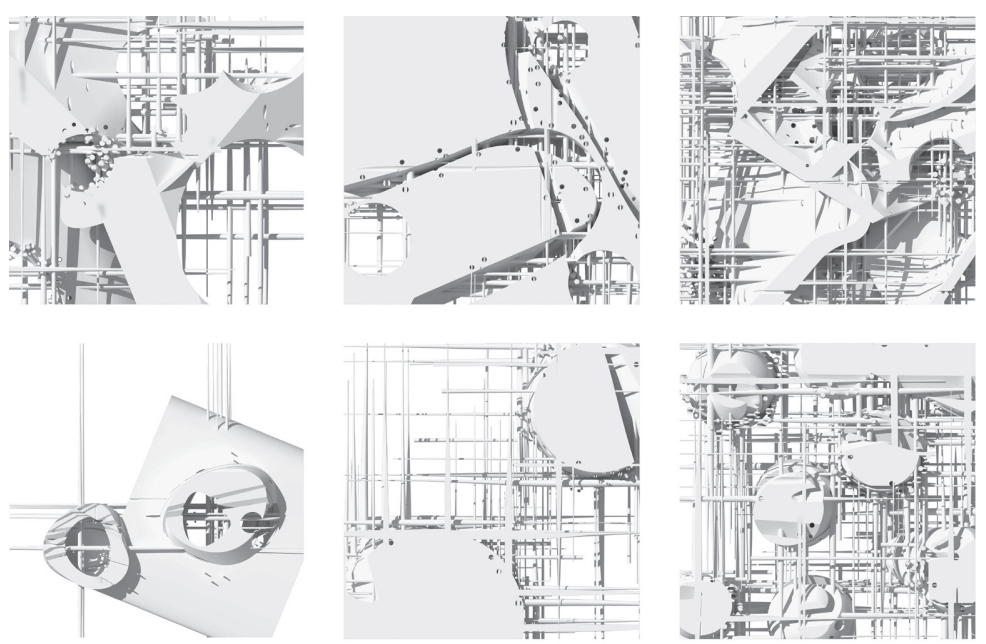

Figura 4

Modelos

combinatorios

logrados a través

de los programas

Rhinoceros y

Grasshopper

Elaboración

propia
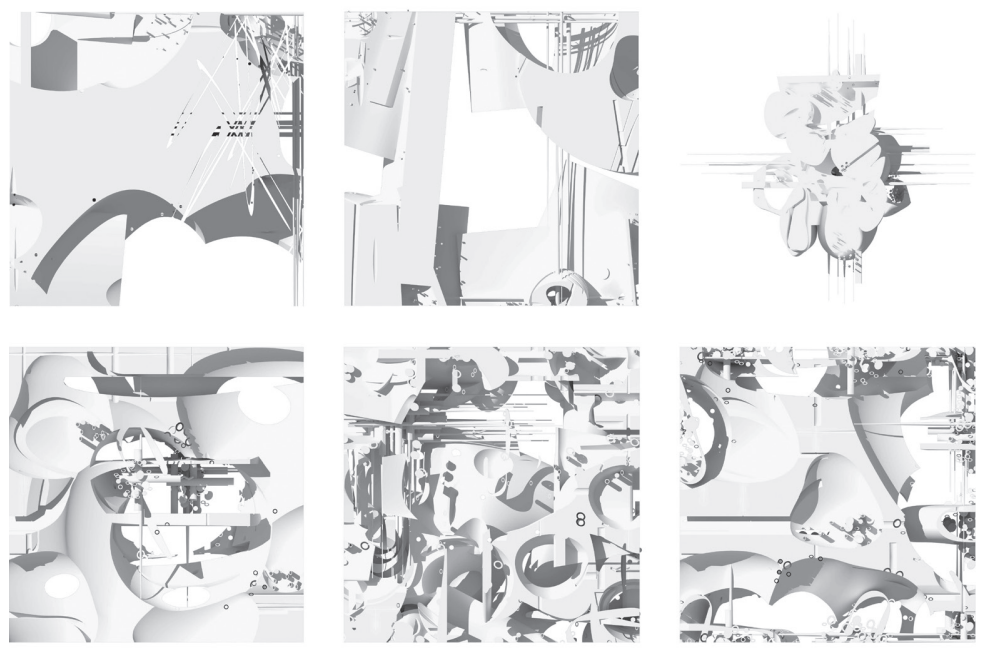

14 Grasshopper es un editor de algoritmos integrado al modelado 3D de Rhinoceros. 


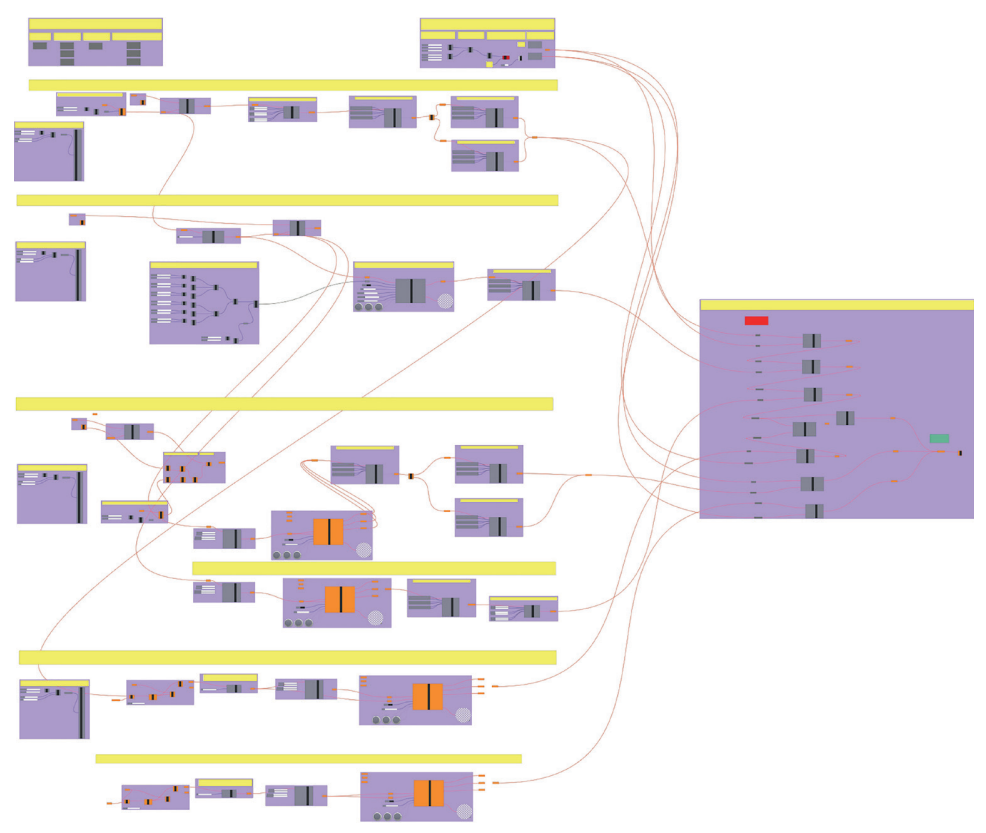

Figura 5.

Algoritmos en

Grasshopper

para producir

modelos combi-

natorios

\section{Elaboración}

propia

\section{EL FUTURO DE LA INTELIGENCIA ARTIFICIAL}

Según la revista The Economist, desde el punto de vista laboral, para el año 2037 existe la tendencia de que las máquinas reemplacen en un $47 \%$ los trabajos que realicen los humanos y se espera que para el año 2067 la mayoría de los trabajos sean autónomos debido a las mejores cualidades que irán desarrollando las máquinas frente a los humanos. En el campo de la arquitectura, el impacto podría verse reflejado en la disminución de la cantidad de proyectistas o personas involucradas en la concepción y desarrollo de proyectos.

Sin embargo, otros especialistas, como el doctor Ismat As, opinan que esta mejora de la inteligencia artificial no debe de ser considerada como una amenaza de suplantación de la máquina respecto a los arquitectos, sino todo lo contrario: entenderla como una gran herramienta por sus beneficios en las diversas etapas del diseño. De igual manera, Ismat As considera que dicho desarrollo puede servir para ampliar la llegada de la arquitectura a personas ajenas a la disciplina, al permitir en un futuro el acceso a programas que contengan lenguajes arquitectónicos, de distintos arquitectos, que puedan ser elegidos, para luego pagar las regalías correspondientes al ser utilizados democratizando, de esta manera, el diseño arquitectónico. 
Bajo otra perspectiva del futuro, centrado en los riesgos de la inteligencia artificial y transhumanista, el filósofo Nick Bostrom ${ }^{15}$, autor del libro Superinteligencia: caminos, peligros, estrategias, concibe que el futuro puede ser estudiado con la misma meticulosidad que el pasado. Parte de la premisa de que no es posible predecir el futuro a corto plazo, aunque es más sencillo imaginarlo en un largo plazo. Bostrom manifiesta que la inteligencia artificial sí puede llegar a representar un peligro en el futuro a largo plazo por la capacidad sin límites que detentaría en los siguientes siglos; puesto que sería capaz de superarse cada día y seguir aprendiendo, inclusive de sus errores, generando con ello que los humanos rivalizaran por primera vez en la historia frente a un competidor más inteligente.

A su vez, Bostrom postula como un posible futuro en varios siglos, alcanzar el siguiente nivel de evolución del ser humano, en el cual la muerte se supera; de esta manera, el ser humano se convertirá, en conjunto con la inteligencia artificial, en una mente súper inteligente que podrá devenir de la conjunción de la mente, memoria y personalidad de una persona con una máquina, siendo posible su propia expansión, a través de un enorme espacio cibernético cognitivo, con billones de mentes digitales.

\section{CONCLUSIONES}

El nivel alcanzado por la evolución y desarrollo de la inteligencia artificial, desde los años 50, nos invita a pensar en un futuro con una mayor evolución de las máquinas, las cuales podrían adquirir e incluso sobrepasar muchas de las cualidades humanas alcanzando un nivel de razonamiento, capacidad de análisis, inteligencia y sensibilidad mayor a la de los humanos. De este modo, ellas nos brindarían recursos para seguir evolucionando.

Dentro de esta evolución, en la disciplina de la arquitectura, el potencial que tiene la inteligencia artificial a un corto o mediano plazo es el de otorgarnos mayores posibilidades de diseño y composición a partir de su capacidad de análisis del terreno y del programa que se le pueda brindar. Esto ayudaría a los proyectistas a tomar mejores decisiones con mayor precisión y sustento de sus trabajos generando con la máquina una arquitectura participativa entre el humano y la inteligencia artificial, más aún cuando se espera una evolución en las próximas décadas.

A este respecto, Richard Sutton, científico informático canadiense, prevé que el sentido de progresión estimado para lograr que la inteligencia artificial alcance un razonamiento similar al humano, es de diez por ciento a que no lo logre nunca y de veinticinco por ciento a que se logrará antes del 2030; mientras que Bostrom y otros científicos le dan un cincuenta por ciento de probabilidades de que esto ocurra antes del 2050.

15 Nick Bostrom (1973) es filósofo, fundador del "Future of Humanity Institute and Programme on the Impacts of Future Technology". 
Por otro lado, al contemplar el potencial de la inteligencia artificial en la arquitectura a largo plazo, podemos apreciar que esta podría ayudarnos a elegir qué tipo de lenguaje arquitectónico (aprendido de los mayores referentes de la arquitectura) es el que quisiéramos aplicar para un nuevo proyecto; o de igual manera, qué tipo de lenguaje arquitectónico es el mejor para ese espacio.

Sin duda, cada etapa de evolución de la inteligencia artificial impactará respecto de las ideas y forma de trabajo de los arquitectos haciéndonos menos participes en algunas tareas, pero permitiéndonos focalizarnos más en otros aspectos, de manera que cada proyecto pueda ir por caminos que no hayan sido imaginados. En suma, impulsando los límites de nuestra propia creatividad e intuición en una búsqueda constante de ser mejores, característica de nuestra humanidad.

Finalmente, no olvidemos que los arquitectos y sus ideas están de paso en un tiempo y espacio determinado; mientras que, las máquinas y sus algoritmos podrían perdurar e influir mucho más, permitiendo que las particularidades humanas replicadas puedan proyectarse a futuras generaciones.

\section{REFERENCIAS}

As, I (17 de setiembre de 2018). Entrevista por Michael J. Crosbie, Common Edge. Recuperado de https://commonedge.org/doom-or-bloom-what-will-artificial -intelligence-mean-for-architecture/

As, I., Pal, S. y Basu, P. (2018). Artificial intelligence in architecture: Generating conceptual design via deep learning. International Journal of Architectural Computing, 16(4), 306-327.

Bostrom, N. (2014). Superintelligence: Path, Dangers, Strategies. Oxford: Oxford University Press.

Galchen, R. (2011). Dream Machine. The New Yorker, 2.

Huang, M., Rust, R. (2018). Artificial Intelligence in Service. Journal of Service Reseach, 21(2), 155-172.

Khatchadourian, R (2015). The doomsday invention. The New Yorker, 23.

Kohler, M (2018). Entrevista por Patricia Guevara, MIT Technology Review en español. Recuperado de https://canalinnovacion.sacyr.com/-/-no-creo-que-la-inteli gencia-artificial-sustituya-a-los-arquitectos-el-diseno-es-mas-social-

Lepore, J (2019). The Robots Caravan: automation, Al, and the coming invasión. The New Yorker.

Llamas, J., M. Lerones, P., Medina, R., Zalama, E. y Gómez-García- Bermejo, J. (2017). Classification of Architectural Heritage Images Using Deep Learning Techniques, Applied Sciences, 7(10), 992. 
Metz, C (2016). The Rise of Artificial Intelligence and the End of Code. Wired. Recuperado de https://www.wired.com/2016/05/google-alpha-go-ai/

Russell, S., Norvig, P. (2009). Artificial Intelligence: A Modern Approach. (tercera edición). India: Pearson Education India.

Valencia, N (2018). Will Automation Affect Architects? ArchDaily. Recuperado de https://www.archdaily.com/885987/will-automation-affect-architects

Yoshimura, Y., Cai, B., Wang, Z. y Ratti, C. (2018). Deep Learning Architect: Classification for Architectural Design through the Eye of Artificial Intelligence. Senseable City Laboratory. Recuperado de https://arxiv.org/pdf/1812.01714.pdf 